Supporting Information (SI) for

\title{
Bilirubin-Coated Radio-Luminescent Particles for Radiation-Induced Photodynamic Therapy
}

Vincenzo J. Pizzutit,§, Dhushyanth Viswanath ${ }^{\dagger} \S$, Sandra E. Torregrosa-Allen ${ }^{\ddagger}, l$, Melanie P. Currie ${ }^{\ddagger, \|,}$ Bennett D. Elzey $y^{\ddagger, \|}$, and You-Yeon Won ${ }^{*,+, \ddagger}$

tDavidson School of Chemical Engineering, ${ }^{\dagger}$ Purdue University Center for Cancer Research, "Department of Comparative Pathobiology, Purdue University, West Lafayette, Indiana 47907, United States

§o-first authors

${ }^{*}$ Corresponding author (Email: yywon@ecn.purdue.edu) 
Figure S1. ${ }^{1} \mathrm{H}$ NMR Spectra for PEG-BR. The PEG-BR product was methanol extracted, dried, and redissolved in DMSO-d6 $(5 \mathrm{mg} / \mathrm{mL})$ for ${ }^{1} \mathrm{H}$ NMR spectroscopy. The peak at $2.5 \mathrm{ppm}$ is due to DMSO-d6, and the peak at $3.25 \mathrm{ppm}$ is due to a trace amount of water. Data confirm that PEG-BR was successfully synthesized.

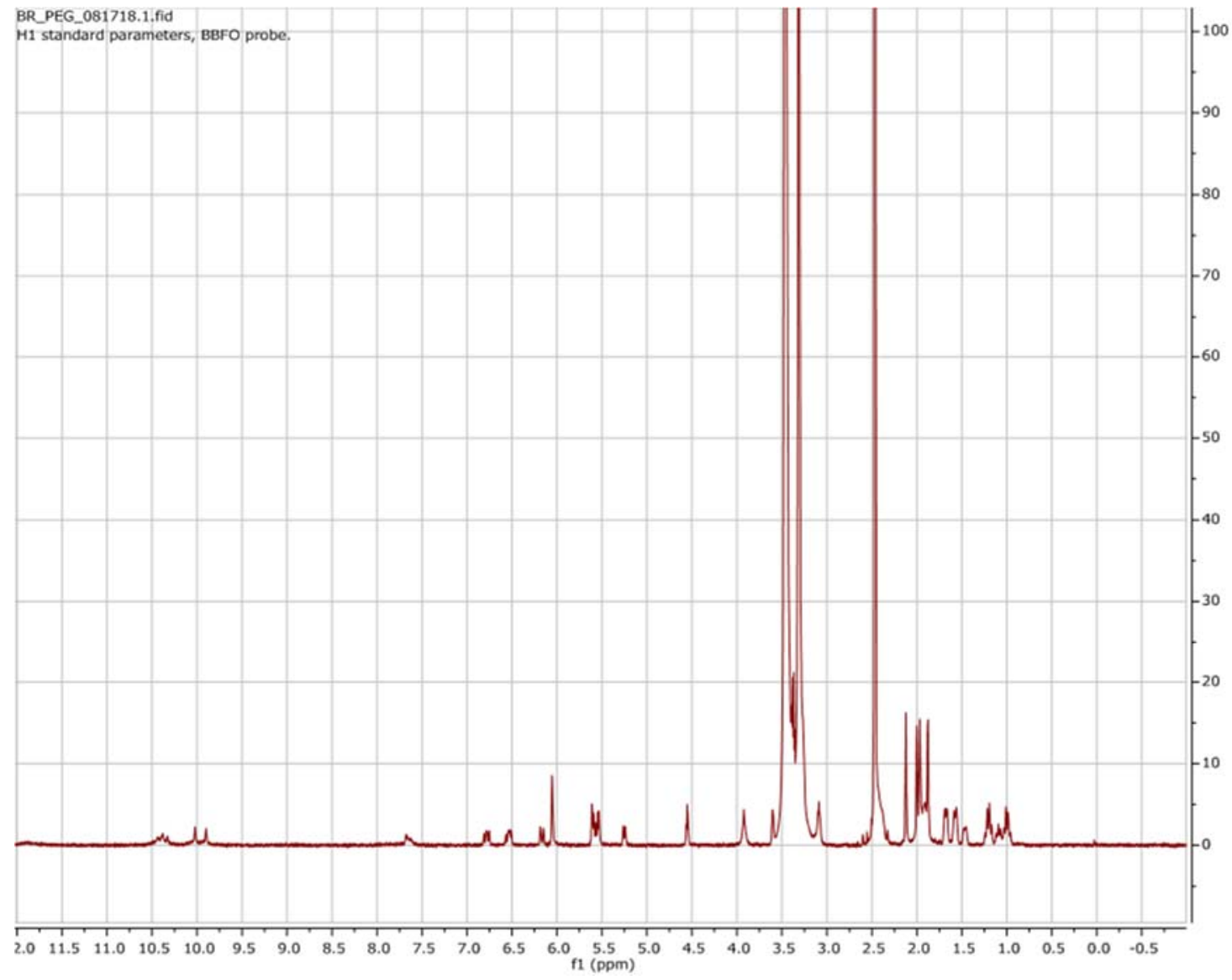


Figure S2. GPC Traces of PEG-BR and Its Precursors. PEG-BR and its precursors, PEG-NH (Laysan $^{-}$ Bio, $2.0 \mathrm{kDa}$ ) and BR (Alfa Aesar), were dissolved in HPLC-grade THF (1 mg/mL) for GPC analysis (filtered with a $450 \mathrm{~nm}$ PTFE filter prior to GPC).

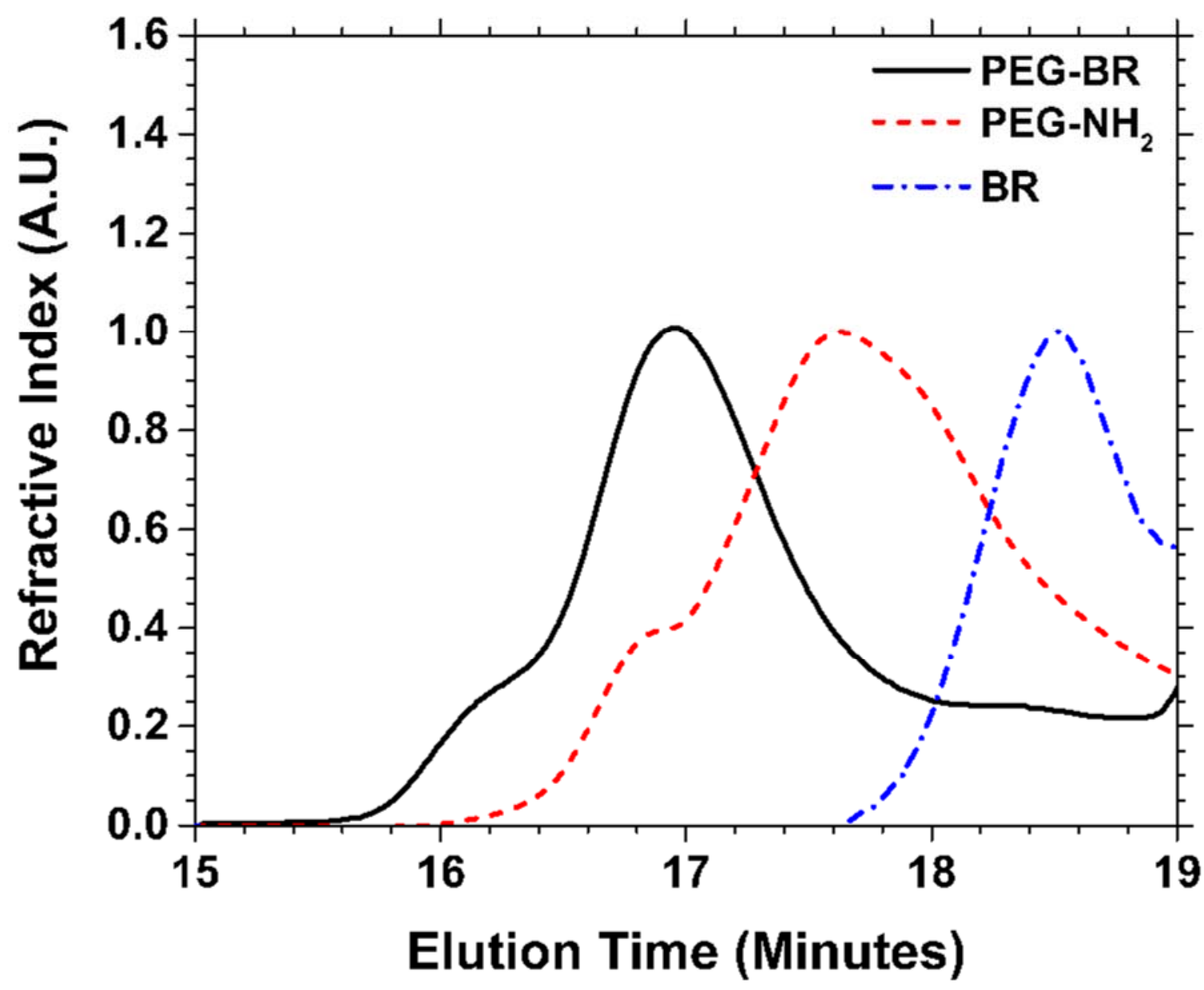


Figure S3. GPC Traces of PEG-BR before and after UV irradiation. PEG-BR NPs (micelles) in PBS were irradiated with UV-A $\left(0.56 \mathrm{~J} / \mathrm{cm}^{2}\right.$ or $\left.61.6 \mathrm{~J} / \mathrm{cm}^{2}\right)$. PEG-BR was extracted from these solutions with $\mathrm{DCM}$, dried, and re-dissolved in HPLC-grade THF $(1 \mathrm{mg} / \mathrm{mL}$ ) for GPC analysis (filtered with a $450 \mathrm{~nm}$ PTFE filter prior to GPC).

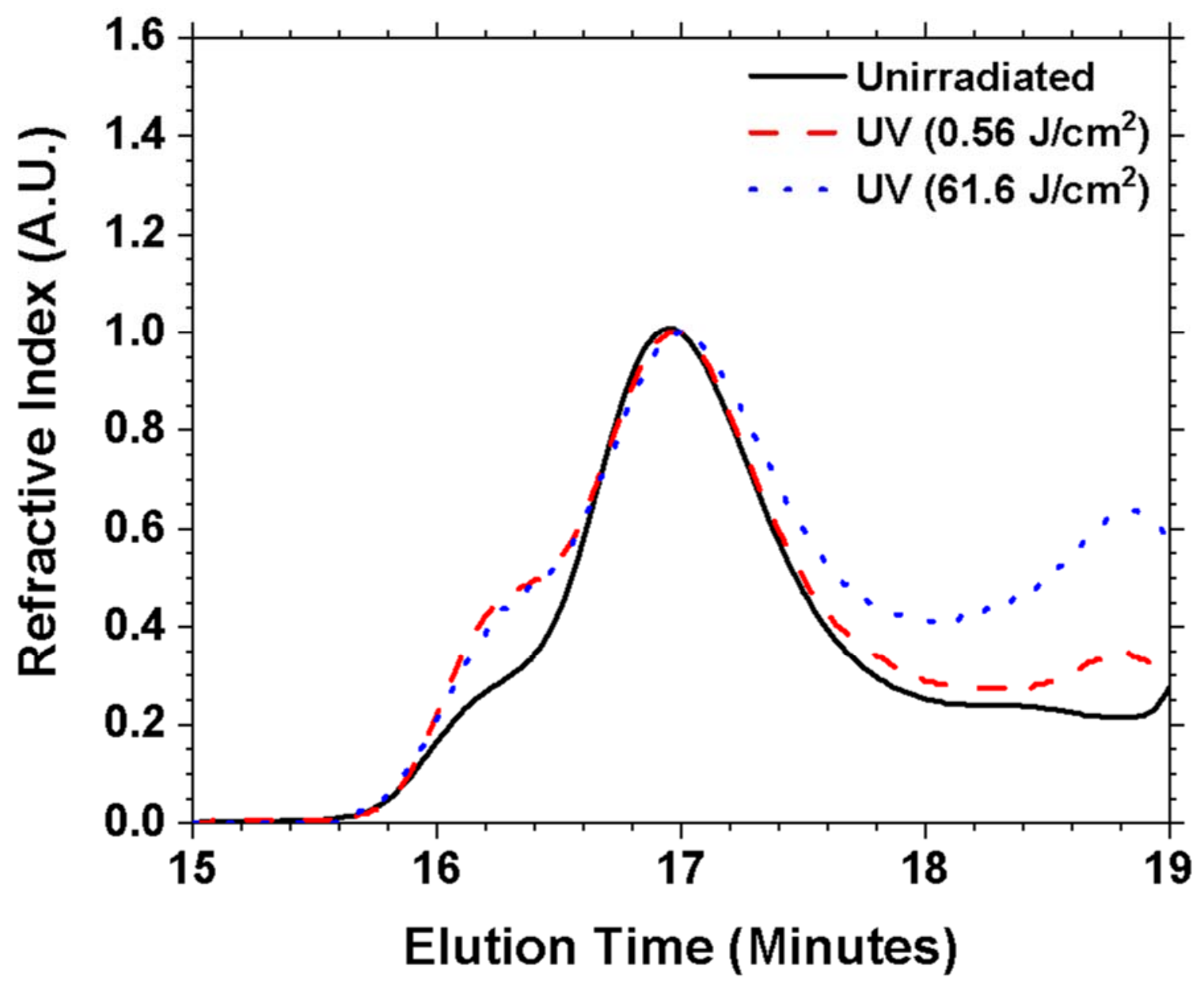


Figure S4. DLS Size Data for UV-A-Irradiated PEG-BR NPs. PEG-BR NPs (micelles) at a concentration of $0.1 \mathrm{mg} / \mathrm{mL}$ in PBS were irradiated with UV-A light (peak emission at $365 \mathrm{~nm}$ ) at a fluence of 0.56 or 61.6 $\mathrm{J} / \mathrm{cm}^{2}$. DLS size measurements were conducted immediately after formulation, and after UV-A exposure. The size of PEG-BR micelles was not influenced by UV-A irradiation. Error bars represent standard deviation $(N=1)$. Note: Experiment conducted using filtered micelles.
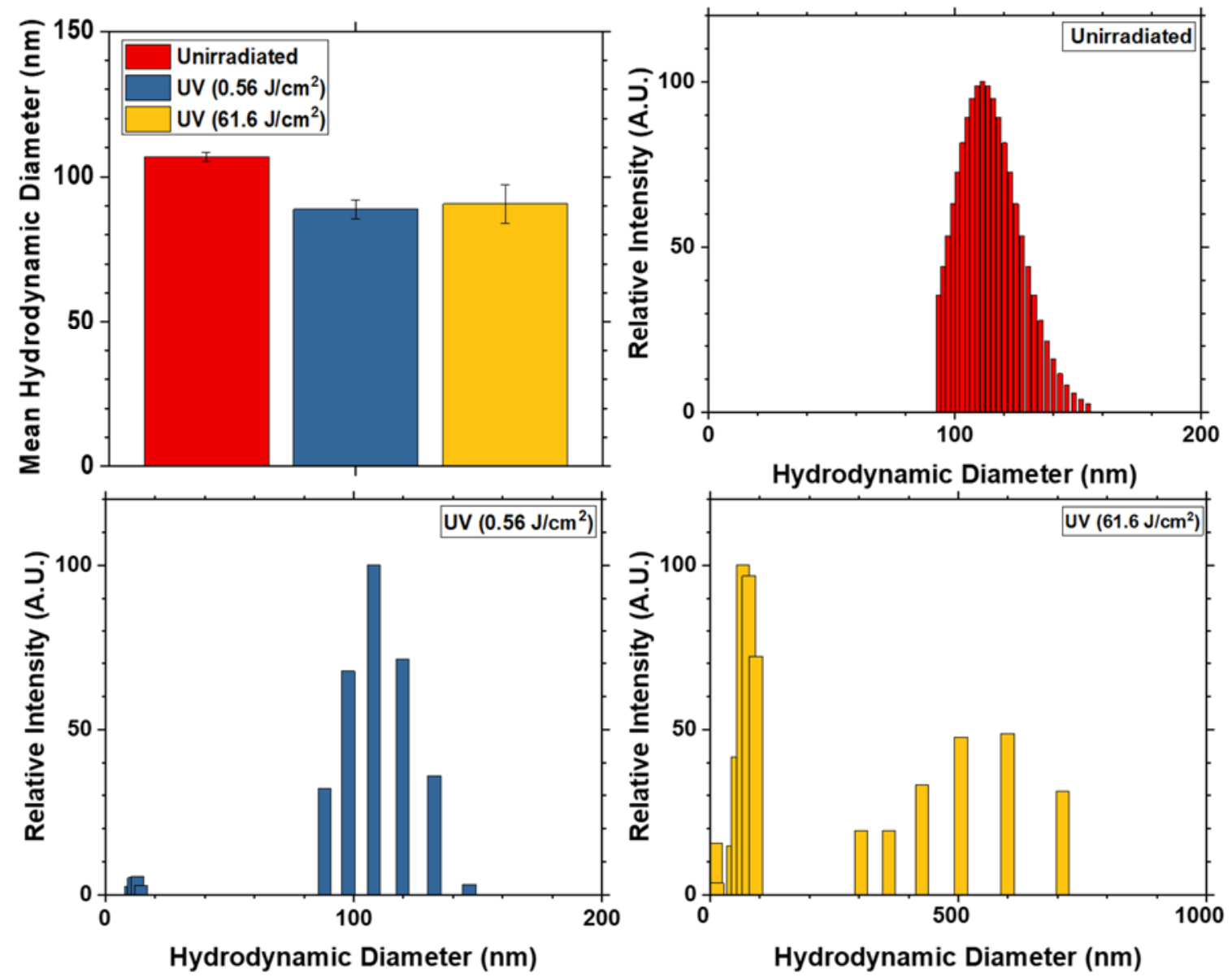
Figure S5. Absorbances and Fluorescences of Irradiated PEG-BR NPs. PEG-BR NPs (micelles) were suspended at $0.1 \mathrm{mg} / \mathrm{mL}$ in PBS. Absorbance (left) and fluorescence (right, excitation wavelength $200 \mathrm{~nm}$ ) measurements were performed using a quartz cuvette with $1 \mathrm{~cm}$ path length at $1 \mathrm{~nm}$ wavelength intervals. PBS was used as the blank reference for absorbance measurements.
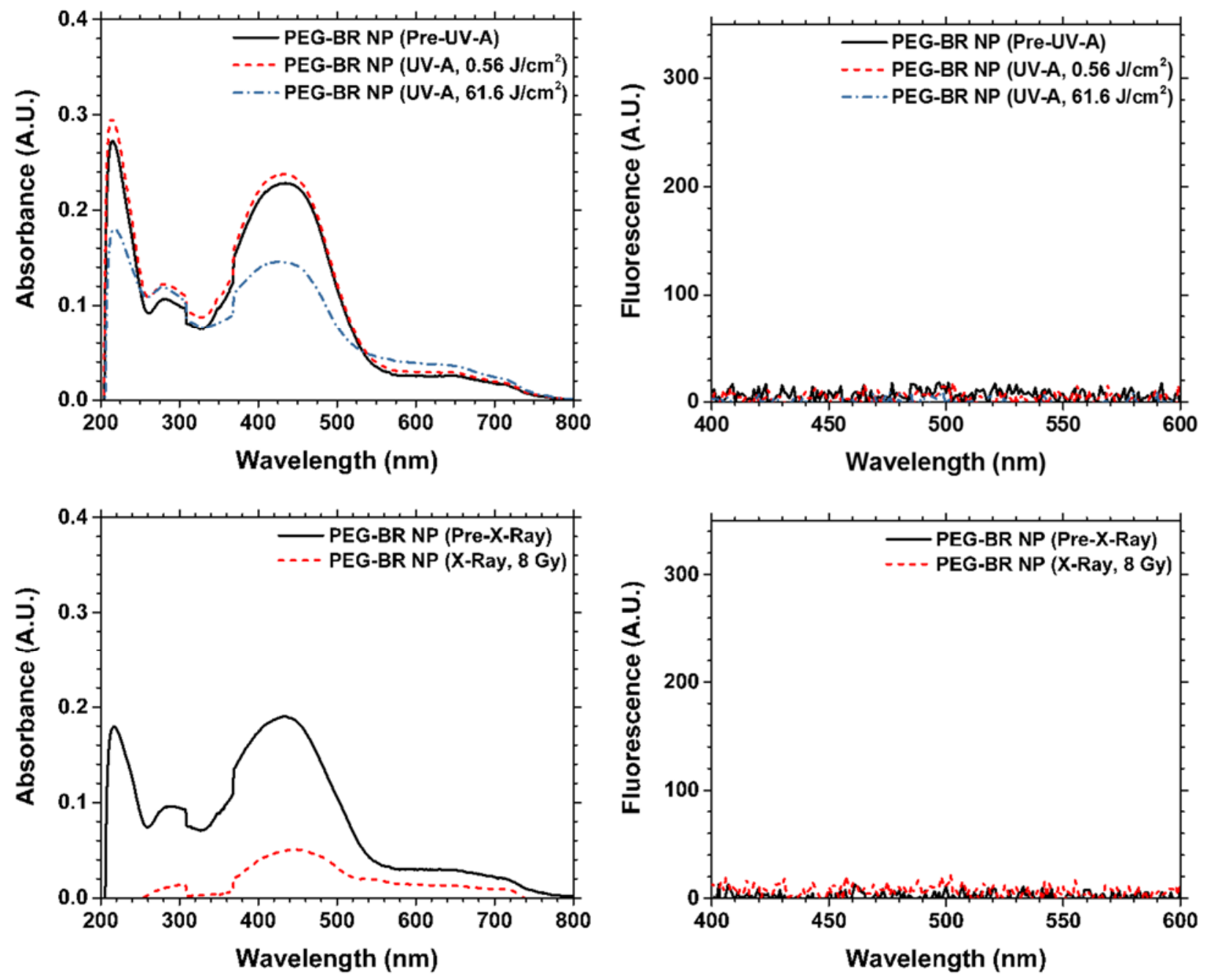
Figure S6. Absorbances and Fluorescences of Irradiated CWO NPs. Uncoated CWO NPs were suspended at $0.1 \mathrm{mg} / \mathrm{mL}$ in PBS. Absorbance (left) and fluorescence (right, excitation wavelength $200 \mathrm{~nm}$ ) measurements were performed using a quartz cuvette with $1 \mathrm{~cm}$ path length at $1 \mathrm{~nm}$ wavelength intervals. PBS was used as the blank reference for absorbance measurements.
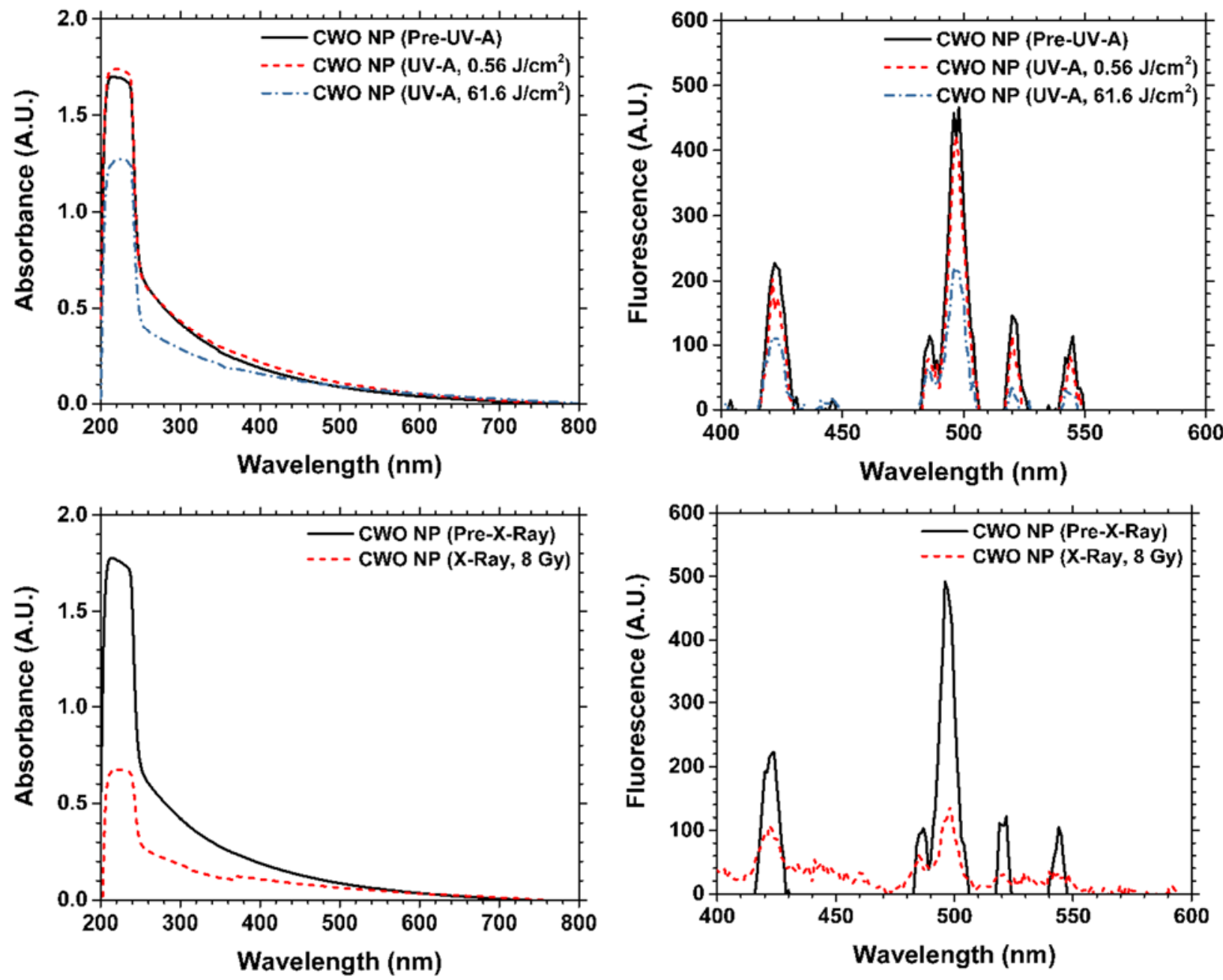
Figure S7. Dot Plots from FACS Analysis. HN31 cells were seeded in T-25 cell culture flasks at a density of $6 \times 10^{5}$ cells/flask. Cells were incubated in a growth medium containing CWO NPs or PEG-BR/CWO NPs at a NP concentration of $0.1 \mathrm{mg} / \mathrm{mL}$ (based on CWO NP concentration) for $4 \mathrm{~h}$ prior to $8 \mathrm{~Gy} \mathrm{X}$-ray exposure. $\mathrm{X}$-ray irradiation was performed at a dose rate of $2 \mathrm{~Gy} / \mathrm{min}$ using a $320 \mathrm{kV}$ X-ray irradiator. Cells were collected via trypsin treatment at $24 \mathrm{~h}$ post irradiation, double stained with FITC Annexin $\mathrm{V}$ and Ethidium Homodimer III, and analyzed on a BD LSRFortessa Cell Analyzer using the FITC and PE laser lines. (A) Signals from cellular debris were first gated out on a 2D dot plot of forward scatter (FSC) vs. side scatter (SSC) intensities (demonstrated for unstained PBS-treated unirradiated cells). (B) Cell doublets were next gated out based on size, i.e., by excluding signals with FSC intensity greater than approximately $2 \times$ median of the FSC intensity for unstained PBS-treated unirradiated cells (demonstrated for (Left) unstained PBStreated unirradiated cells and (Right) unstained PEG-BR/CWO NP-treated unirradiated cells). This identical gating criterion was applied to all stained/unstained, CWO NP and PEG-BR/CWO NP-treated, irradiated/unirradiated subgroups. (C) On top of (A) and (B) above, non-HN31 signals were gated out based on complexity by comparing FITC vs. SSC intensity plots for stained/unstained, CWO NP and PEGBR/CWO NP-treated, irradiated/unirradiated subgroups with the unstained PBS-treated unirradiated control (demonstrated for (Left) unstained PBS-treated unirradiated cells and (Right) unstained PEG-BR/CWO NPtreated unirradiated cells). (D) Within the resultant cell population, FITC/PE-negative and FITC/PE-positive subpopulations were identified based on the quadrant criteria established using data from unstained and single-stained PBS-treated X-ray-irradiated controls (the same quadrant criteria were applied for all subgroups). The different quadrants represent different cell states: Q1 (upper left) = early apoptosis, Q2 (upper right) = apoptosis, Q3 (lower left) = live, Q4 (lower right) = necrosis. See Figure 12 for actual population distributions. (E) The validity of this procedure was tested by examining data from unstained PEG-BR/CWO NP-treated unirradiated cells. As shown in (E), all false signals from PEG-BR which were originally present in the FITC and/or PE-positive quadrants ( $B$ and $C$ ) have been completely gated out using the above procedure.

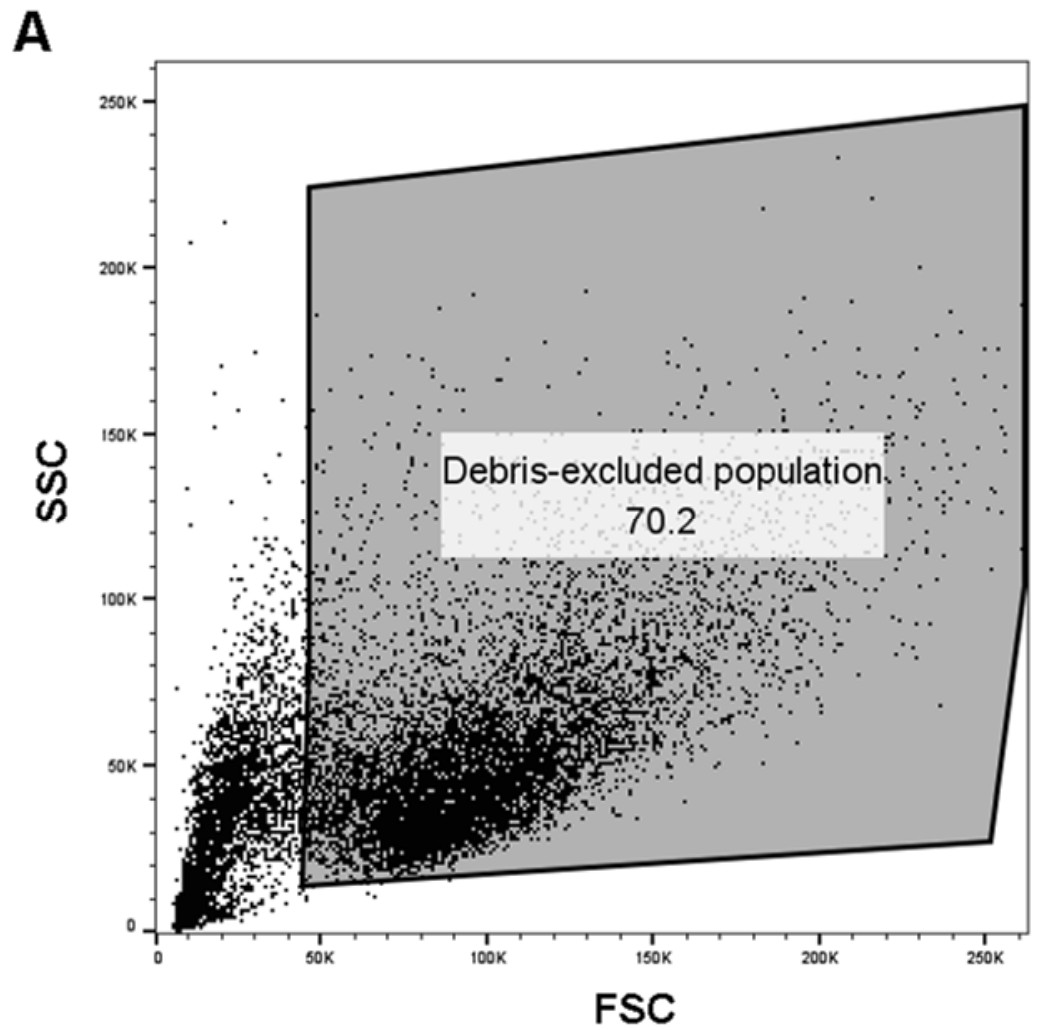


B

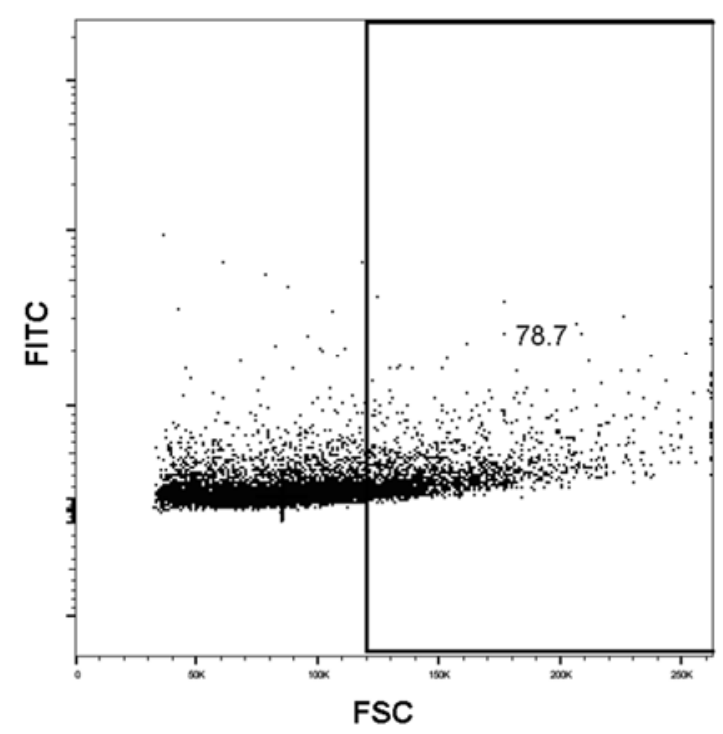

C

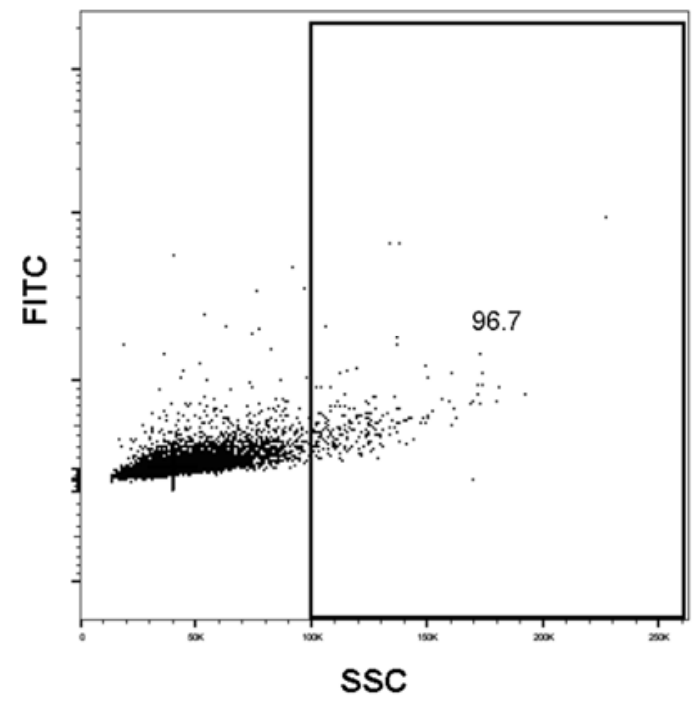

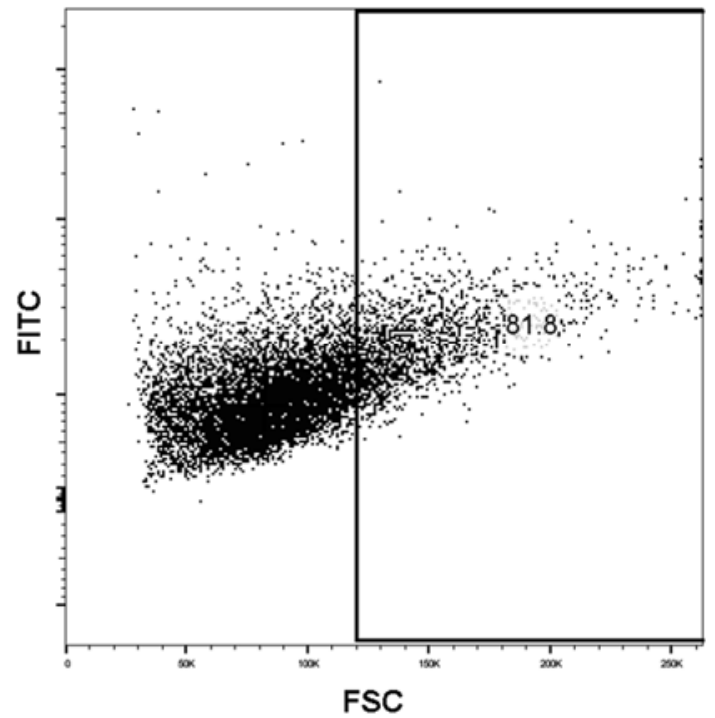

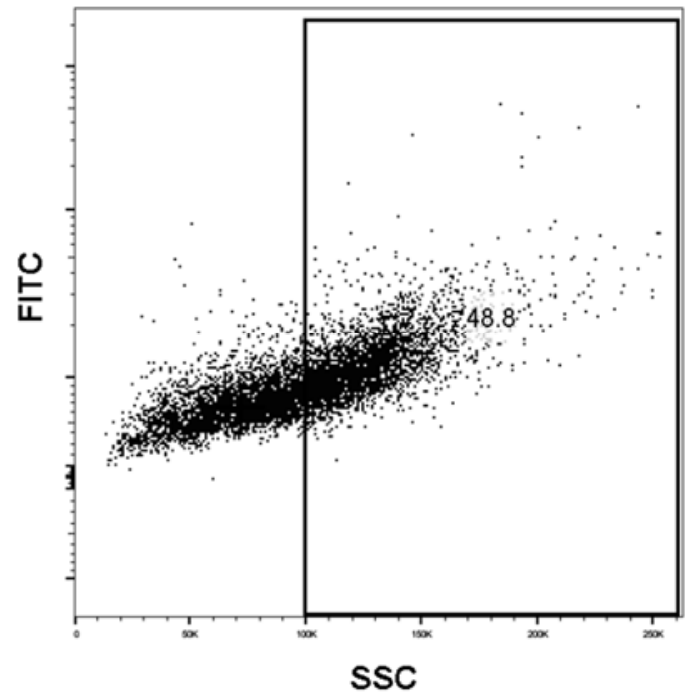


D

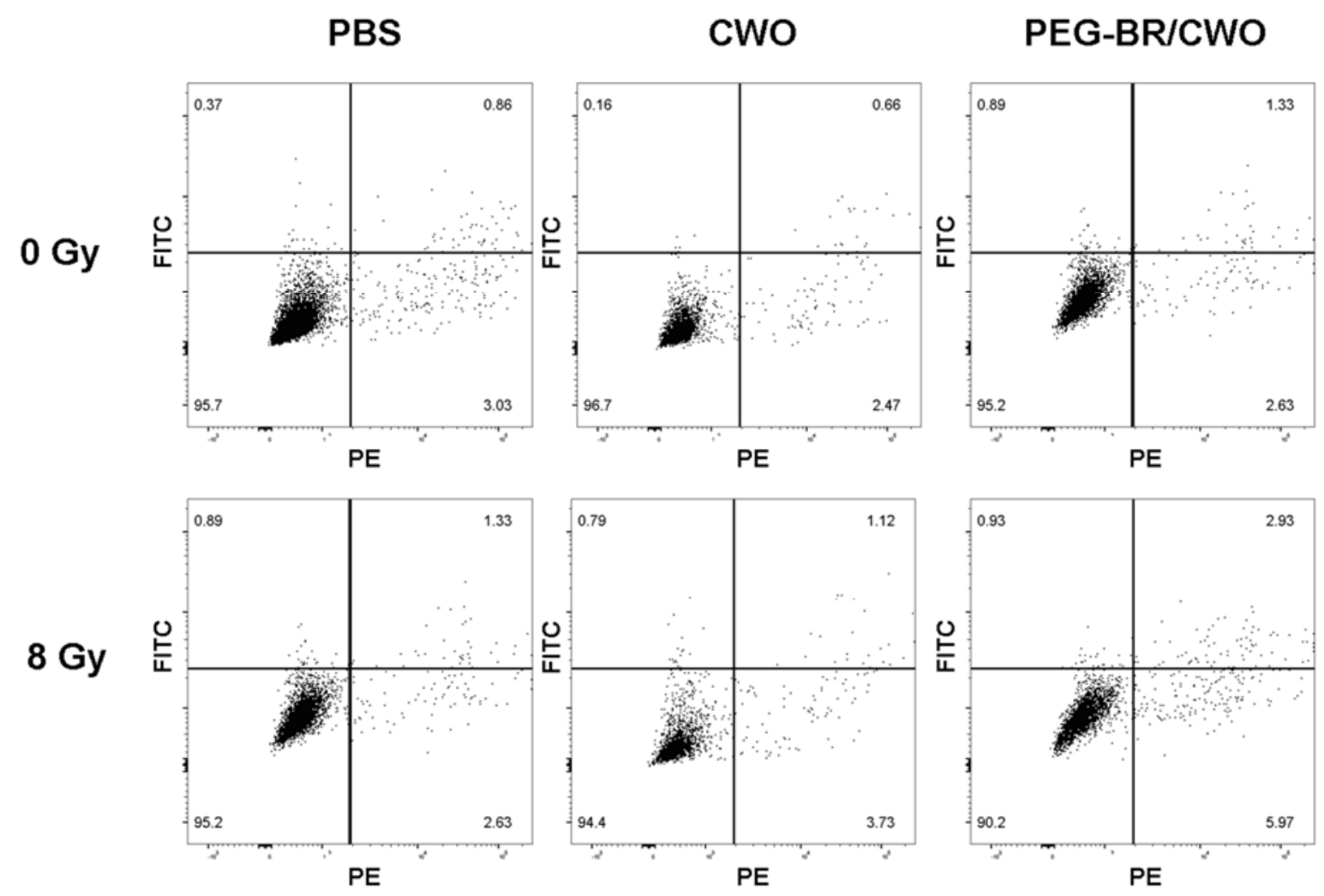

E

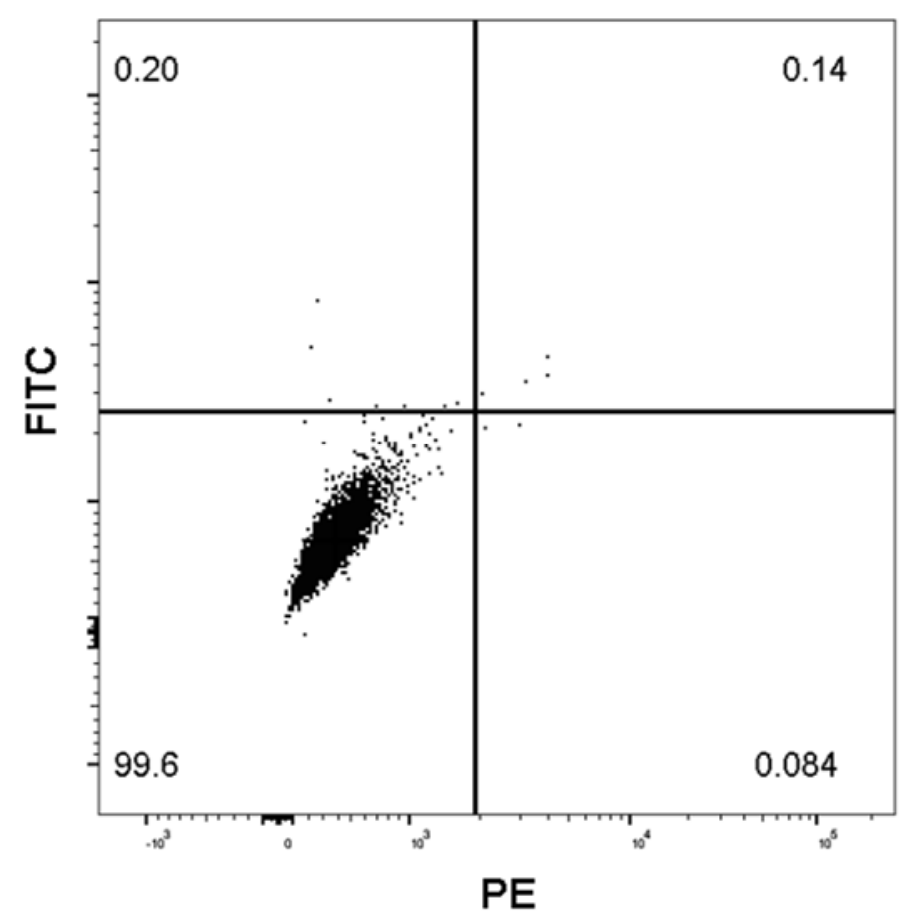


Figure S8. Tumor Doubling Time Estimation. Tumor volume data from unirradiated mouse groups at early time points were fit to the power-law expression, $\frac{v}{V_{o}}=2^{t_{d} / t} ; V_{0}=100$ and $120 \mathrm{~mm}^{3}$ for the 4 Gy (left; Figure 12) and $8 \mathrm{~Gy}$ (right; Figure 14) experiments, respectively. The resultant $t_{d}$ values are given in Table 1.
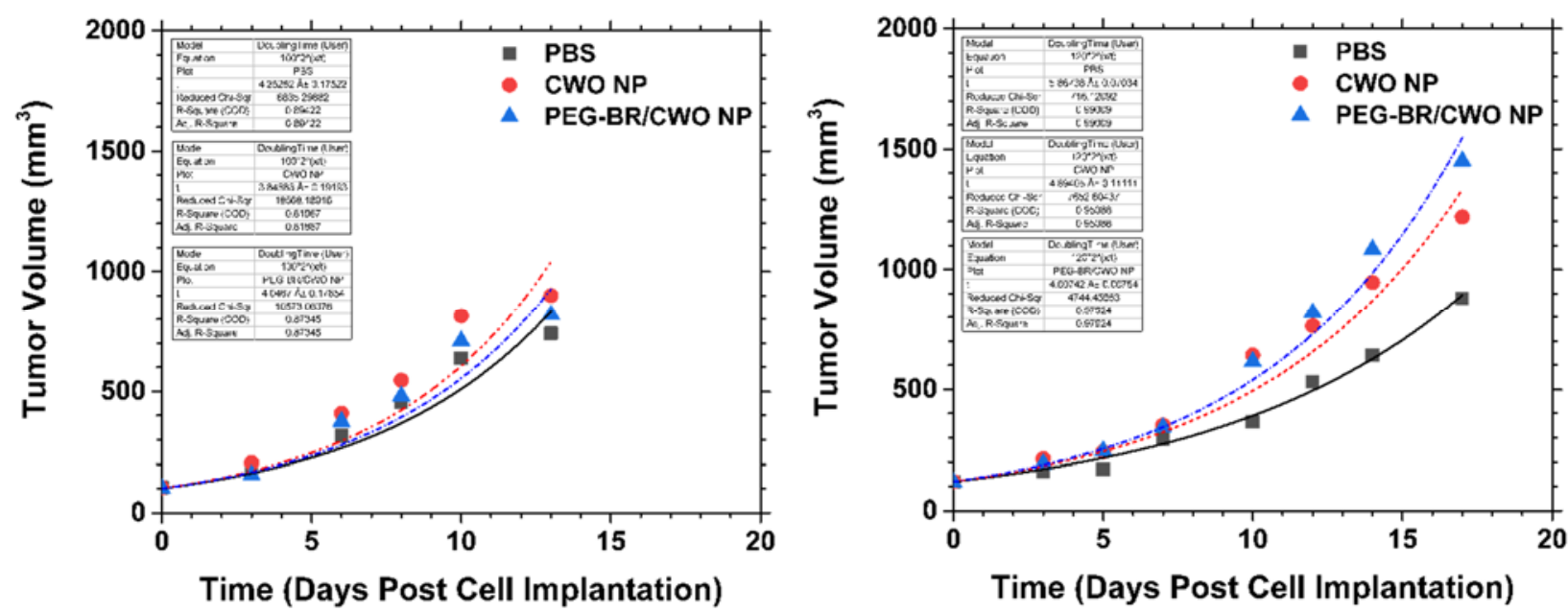
Figure S9. Mouse Body Weight Trends. Mouse body weights recorded as a function of time following first NP injection for mice tested in the study shown in Figures 13 and 14.
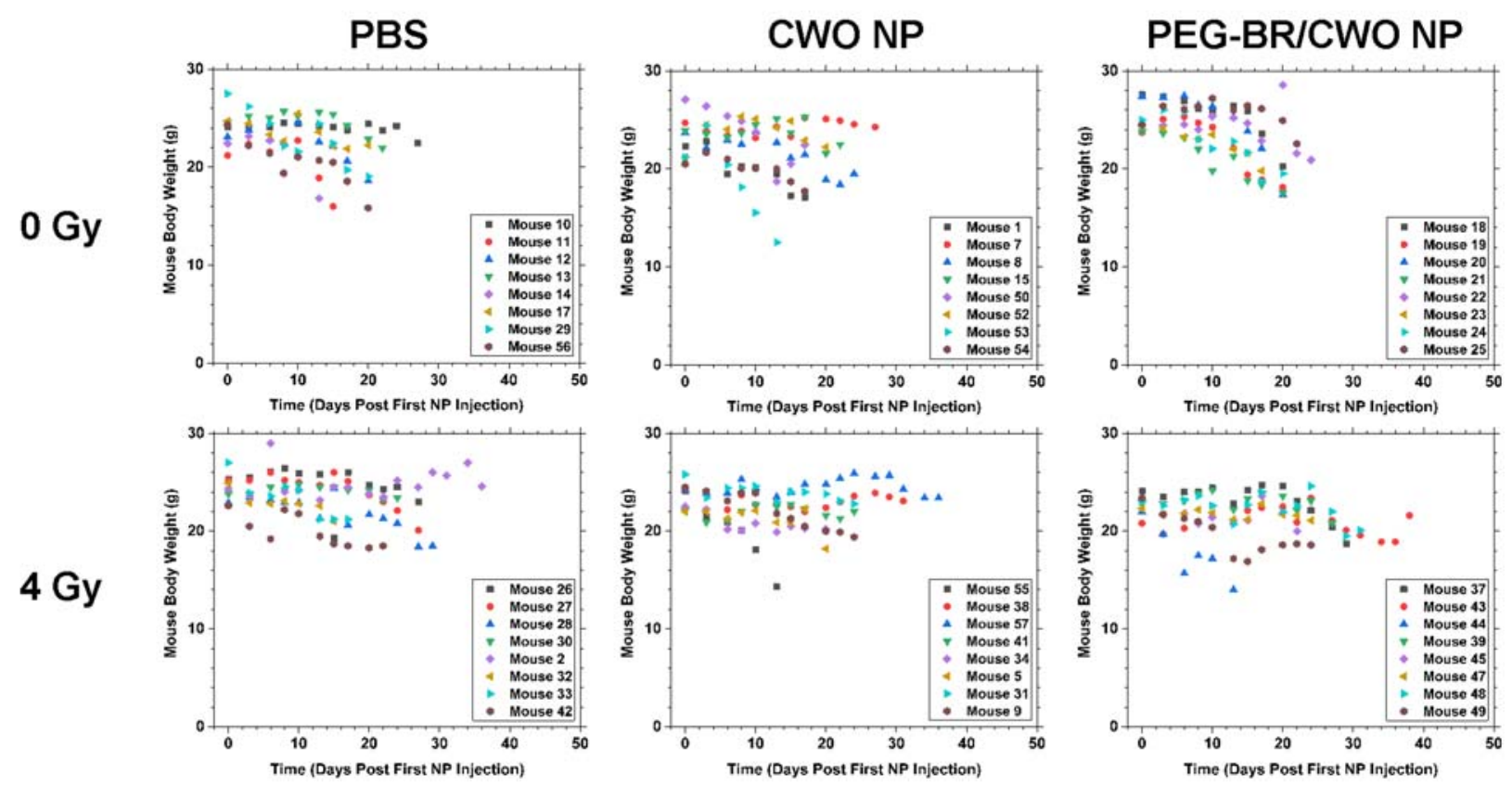
Figure S10. Mouse Body Weight Trends. Mouse body weights recorded as a function of time following first NP injection for mice tested in the study shown in Figures 15 and 16.
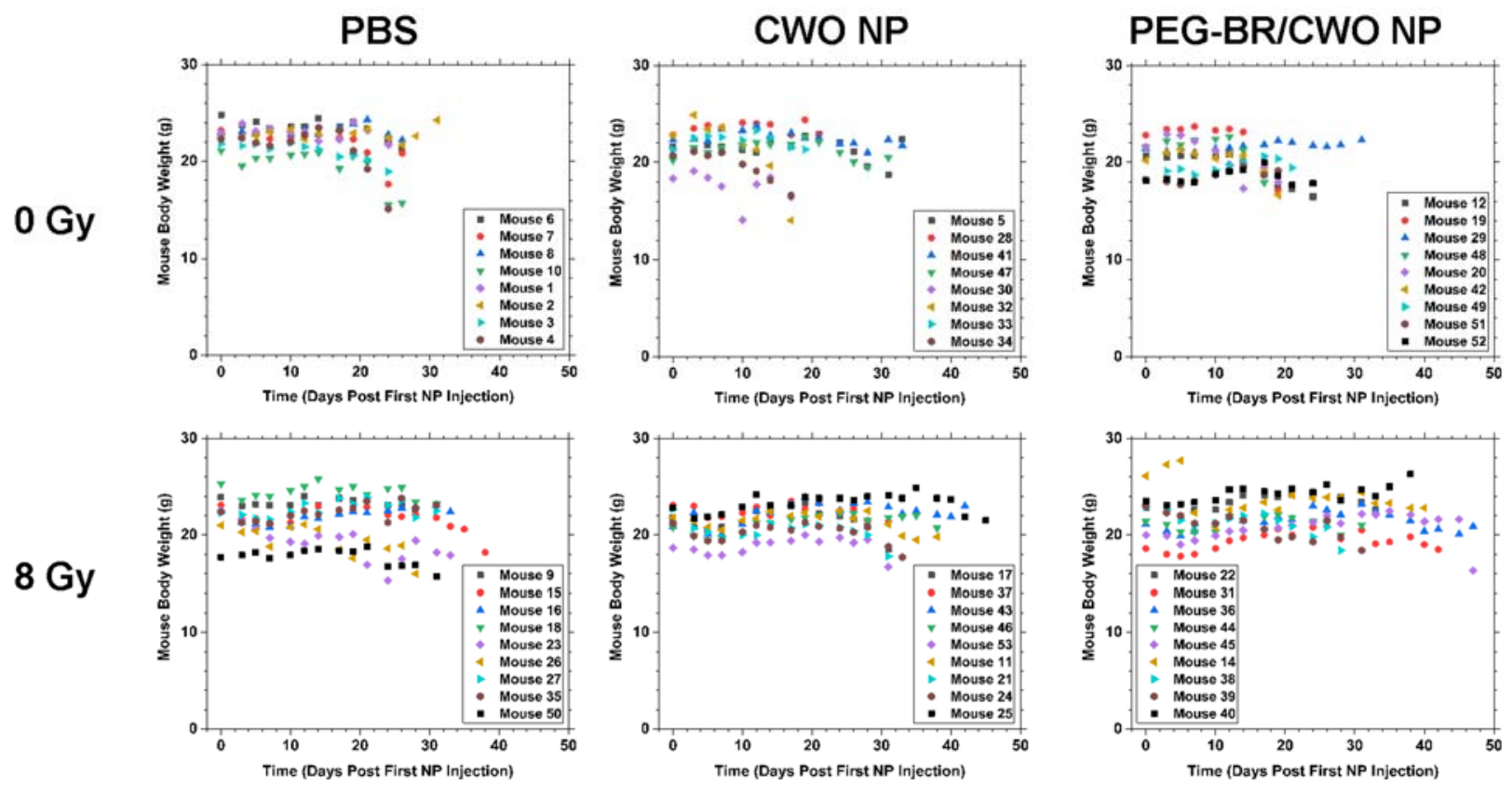